\title{
Convergence of SPH and AMR simulations
}

\author{
David A. Hubber ${ }^{1,2}$, Sam A. E. G. Falle ${ }^{3}$ and Simon P. Goodwin ${ }^{1}$ \\ ${ }^{1}$ Department of Physics and Astronomy, University of Sheffield, \\ Hicks Building, Hounsfield Road, Sheffield, S3 7RH, UK \\ ${ }^{2}$ School of Physics and Astronomy, University of Leeds, Leeds, LS2 9JT, UK \\ ${ }^{3}$ Department of Applied Mathematics, University of Leeds, Leeds, LS2 9JT, UK
}

\begin{abstract}
We present the first results of a large suite of convergence tests between Adaptive Mesh Refinement (AMR) Finite Difference Hydrodynamics and Smoothed Particle Hydrodynamics (SPH) simulations of the non-linear thin shell instability and the Kelvin-Helmholtz instability. We find that the two methods converge in the limit of high resolution and accuracy. AMR and SPH simulations of the non-linear thin shell instability converge with each other with standard algorithms and parameters. The Kelvin-Helmholtz instability in SPH requires both an artificial conductivity term and a kernel with larger compact support and more neighbours (e.g. the quintic kernel) in order converge with AMR. For purely hydrodynamical problems, SPH simulations take an order of magnitude longer than the grid code when converged.
\end{abstract}

Keywords. hydrodynamics, methods: numerical

\section{Introduction}

Most simulations of self-gravitating hydrodynamics in astrophysics are performed using either Adaptive Mesh Refinement (AMR) Finite Difference hydrodynamics or Smoothed Particle Hydrodynamics (SPH; Lucy 1997; Gingold \& Monaghan 1977). Both methods attempt to solve the fluid equations using very different paradigms, each with its own advantages and disadvantages (e.g. grid vs. particles, or Eulerian vs. Lagrangian). Ultimately they should both give the same result in the limit of high resolution and accuracy. Only in the limit of low resolution and accuracy should their limitations become important enough to warrant special attention.

One particular problem with SPH that has been highlighted in recent years is its inability to correctly resolve mixing with particular attention given to the Kelvin-Helmholtz instability (Agertz et al. 2007). As well as this problem, there are other examples where SPH and AMR may disagree. We have begun a widespread suite of tests using both $\mathrm{SPH}$ and AMR in order to determine i) if SPH and AMR give the same results at high resolution and accuracy; ii) at what resolution(s) SPH and AMR begin to diverge and iii) determine how SPH and AMR behave when under-resolved. We present here the first results of simulations of the non-linear thin-shell instability and the Kelvin-Helmholtz instability using the SPH code SEREN (Hubber et al. 2010; in press) SPH code and the AMR-MHD grid code MG (van Loo, Falle \& Hartquist 2006).

\section{Non-linear thin-shell instability}

The non-linear thin-shell instability (hereafter NTSI; Vishniac 1994) occurs when two colliding streams of gas form a shock along a non-planar boundary. If the interface between the two flows is some form of sinusoidal boundary, then this shape can effectively 'funnel' material towards the extrema of the sinusoid. This leads to the growth of density enhancements as more material flows into the shock. We model the NTSI with two 

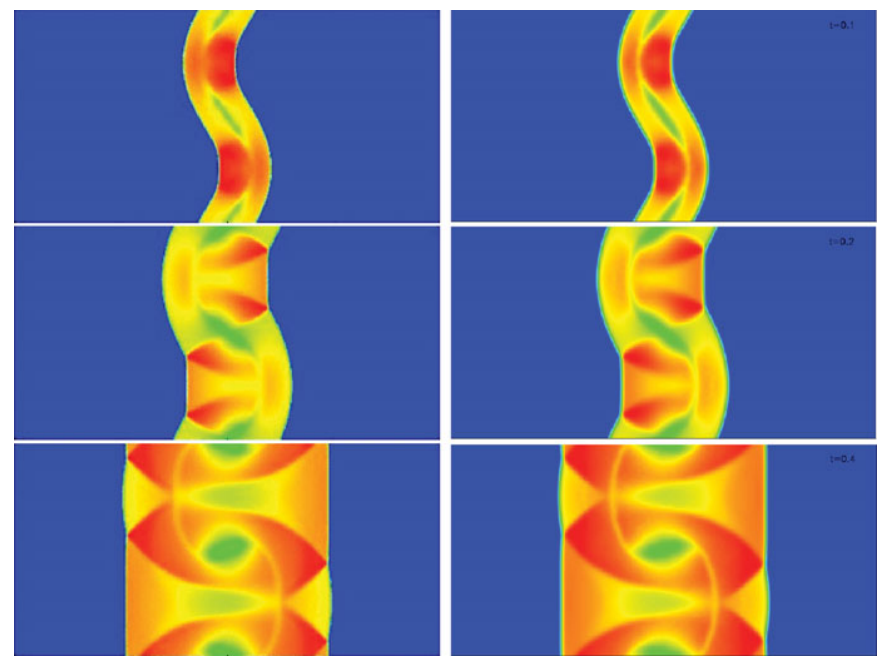

Figure 1. The development of the non-linear thin-shell instability at times $t=0.1$ (top rown), $t=0.2$ (middle row) and $t=0.4$ (bottom row) modelled with MG (left-hand column) and SEREN (right-hand column) in the region $-1<x<1,0<y<1$. Dark colours indicate the pre-shock density and lighter shades the higher-density shocked regions.

uniform density gas flows with the same initial density $(\rho=1)$, constant pressure $P=1$ and ratio of specific heats $\gamma=5 / 3$. The initial velocity profile is

$$
v_{x}(x, y)= \begin{cases}+2 & x<A \sin (k y) \\ -2 & x>A \sin (k y)\end{cases}
$$

where $A=0.1, k=2 \pi / \lambda, \lambda=1$ and $v_{y}=0$ everywhere initially. The computational domain extends between the limits $-3<x<3$ and $0<y<1$ with open boundaries in the $\mathrm{x}$-dimension and periodic boundaries in the $\mathrm{y}$-dimension. We follow the evolution of the instability until $t=0.4$ using MG and SEREN. Both codes use standard algorithms and parameters for this test. The resolution of the grid simulation is $1200 \times 200$ and the number of SPH particles is 240,000, initially placed on a uniform lattice.

In Figure 1, the AMR results (left-hand column) and the SPH results (right-hand column) are shown at times $t=0.1, t=0.2$ and $t=0.4$. This is beyond the growth of the initial density enhancements and towards the generation of more complicated density structures. It can be seen in Figure 1 at $t=0.1$ that the growth of the initial density perturbation still closely follows the initial sinusoidal boundary. At later times, the flow pattern becomes more complex with a more elongated density structure forming. The AMR and SPH results are nearly identical with only small noticable deviations, possibly due to smoothing in SPH. We note that the SPH simulations are performed with the standard M4 kernel (Monaghan \& Lattanzio 1985) and without any additional algorithms such as artificial conductivity (e.g. Price 2008).

\section{Kelvin-Helmholtz instability}

The Kelvin-Helmholtz instability (hereafter KHI) is a classical hydrodynamical instability generated at the boundary between two shearing fluids which leads to vorticity and mixing between the two fluids. Analysis of the linear growth is given in many classical textbooks and papers (e.g. Chandrasekhar 1961; Junk et al. 2010). We model a 2 : 1 density contrast following Agertz et al. 2007 and other authors. The two fluids are separated 

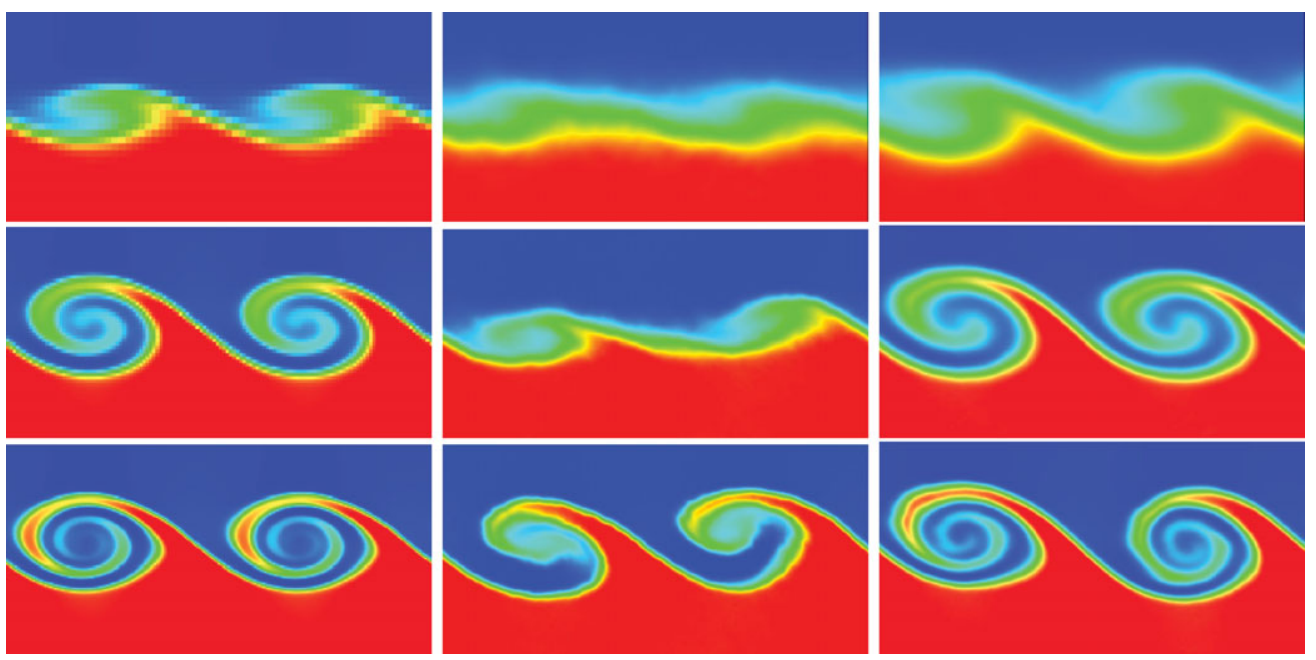

Figure 2. The Kelvin-Helmholtz instability for a 2:1 density contrast at a time $t=2$ modelled with MG (left-hand column), SEREN with the M4 kernel (middle column) and SEREN with the quintic kernel (right-hand column). The resolution of the grid simulation is $64 \times 32$ (top row), $128 \times 64$ (middle row) and $256 \times 128$ (bottom row). The no. of particles in the SPH simulations are 3,274 (top row), 24, 484 (middle row) and 97,936 (bottom row).

along the $\mathrm{x}$-axis and have a $\mathrm{x}$-velocity shear, but are in pressure balance with $P=2.5$. The ratio of specific heats is $\gamma=7 / 5$. Fluid $1(y>0)$ has a density $\rho_{1}=1$ and x-velocity $v_{x}=0.5$. Fluid $2(y<0)$ has a density $\rho_{2}=2$ and x-velocity $v_{x}=-0.5$. The velocity perturbation in the $\mathrm{y}$-direction with wavelngth $\lambda=0.5$ is given by

$$
v_{y}(x, y)= \begin{cases}A \sin (k x) \exp (-k y) & y>0 \\ A \sin (k x) \exp (k y) & y<0\end{cases}
$$

where $k=2 \pi / \lambda$. The computational domain is $-0.5<x<0.5$ and $-0.25<y<0.25$ with periodic and mirror boundaries in the $\mathrm{x}$ - and $\mathrm{y}$-dimensions respectively. We follow the evolution of the KHI until a time of $t=2$ using both MG and SEREN, beyond the linear growth of the instability and into the non-linear regime where vorticity develops.

Figure 2 shows the KHI for AMR (column 1), SPH with the M4 kernel (column 2; Monaghan \& Lattanzio 1985) and SPH with the quintic kernel (column 3; Morris 1996). For the AMR simulations, the resolution of each row from top to bottom is $64 \times 32,128 \times 64$ and $256 \times 128$. For the SPH simulations, the number of particles, from top to bottom, and are 3,274, 24, 484 and 97,936. Figure 2 shows that these choices of resolution give very similar results between the AMR simulations and SPH simulations with the quintic kernel, For the lowest resolution (Figure 2, row 1), the two layers only just start to mix together, but there is insufficient resolution for a true vortex to be modelled. As we increase the resolution in both cases (Figure 2, rows 2 and 3), we can see the two methods develop in a similar way, with more vorticity in the spiral patterns being realised.

The SPH simulations with the M4 kernel (middle row) do not agree very well with the AMR simulations in terms of the development of the vorticity. As we increase the resolution, the results with the M4 kernel improve somewhat, but even for very high resolution, they do not agree very well with the grid code at lower resolution. It should be noted that the simulations with the M4 and quintic kernels contain exactly the same numbers of particles. However, since the quintic kernel has a larger compact support $(3 h$ instead of $2 h$ ), its formal resolution is lower. However, it has higher accuracy than 
the M4 kernel since the larger average number of SPH neighbours means a reduction in particle noise in the SPH summation approximation. Other authors have noted that they require larger neighbour numbers to reduce particle noise and other summation errors (e.g. Read, Hayfield \& Agertz 2010).

\section{Discussion and Future work}

Our simulations of the NTSI and the KHI have shown that AMR and SPH, given enough resolution and accuracy, can show very good agreement in hydrodynamical problems with complex flow patterns. These simulations are only the first in a larger planned suite of convergence tests between SPH and AMR codes, including the Rayleigh-Taylor instability, radiative shocks, advection of polytropes, orbiting binary polytropes.

It is noticeable in our simulations that the NTSI simulations converge very well with standard options and parameters, whereas the SPH KHI required additional algorithms or modifications (e.g. artificial conductivity) plus more neighbours. One principle difference between the two cases is the NTSI is seeded by a large scale, super-sonic perturbation where particle noise and errors are not important, whereas the KHI is the growth of a seeded, low-amplitude velocity perturbation where noise and errors can corrupt the instability early on before it can grow. The accuracy of the SPH method (controlled somewhat by the number of neighbours) required to converge on the same results as the AMR code is therefore dependent somewhat on the problem studied. This is clear from the KHI convergence tests where the M4 kernel does not appear to converge no matter how high the resolution. An important consequence of this is SPH convergence studies should consider varying both the total particle number and neighbour number.

One important practical implication of these convergence tests is that where convergence is achieved, the run times of the SPH simulations are more than an order of magnitude longer than the AMR simulations. This suggests that SPH is not the optimal method for pure-hydrodynamical simulations. The only principal advantage that SPH maintains over grid codes in such scenarios is its Lagrangian nature, which may be important when large advection velocities are present (e.g. Springel 2010). However, preliminary simulations of tests involving self-gravity (e.g. polytrope tests) suggest that the disparity in run-times of astrophysics simulations will not be so great (see also, Federrath et al. 2010).

\section{References}

Agertz, O., Moore, B., Stadel, J., et al., 2007, MNRAS, 380, 963

Chandrasekhar, S., 1961, 'Hydrodynamic and Hydromagnetic Stability', Oxford University Press Federrath, C., Banerjee, R., Clark, P. C., Klessen, R. S., 2010, AJ, 713, 269

Gingold, R. A., \& Monaghan, J. J., 1977, MNRAS, 181, 375

Hubber, D. A., Batty, C. P., McLeod, A., Whitworth, A. P., 2010, A\&\&A, submitted

Lucy, L., 1977, AJ, 82, 1013

Junk, V., Walch, S., Heitsch, F., et al., 2010, MNRAS, submitted

Monaghan, J. J., Lattanzio, J. C., 1985, A\& A, 149, 135

Morris, J. P., 1996, PhD Thesis - 'Analysis of Smoothed Particle Hydrodynamics with Applications', Monash University

Price, D. J., 2008, JCoPh, 227, 10040

Read, J. I., Hayfield, T., Agertz, O., 2010, MNRAS, 405, 1513

Springel, V., 2010, MNRAS, 401, 791

van Loo, S., Falle, S. A. E. G., Hartquist, T. W., 2006, MNRAS, 370, 975

Vishniac, E. T., 1994, ApJ, 428, 186 